\title{
«... und bei den Patienten unwiderrufliche Schäden»
}

\section{Daniel Lüthi}

Freier Journalist und Fotograf, Medientrainer, Bern

"Globalbudget im Gesundheitswesen»: In der Schweiz ist das Thema noch jung. Anfang Jahr hat sich die Sozial- und Gesundheitskommission des Nationalrates positiv dazu geäussert. Zuvor hatte sich FmCh-Präsident Josef E. Brandenberg bereits in Holland und Deutschland informieren lassen, die das System seit Jahren kennen. «Bei mir läuteten sofort die Alarmglocken», sagte er in Luzern. Als Reaktion auf diese «böse Vorahnung» griff die fmCh dort das Thema an einem Symposium auf - dieses liess dem Globalbudget nicht ein einziges positives Argument.

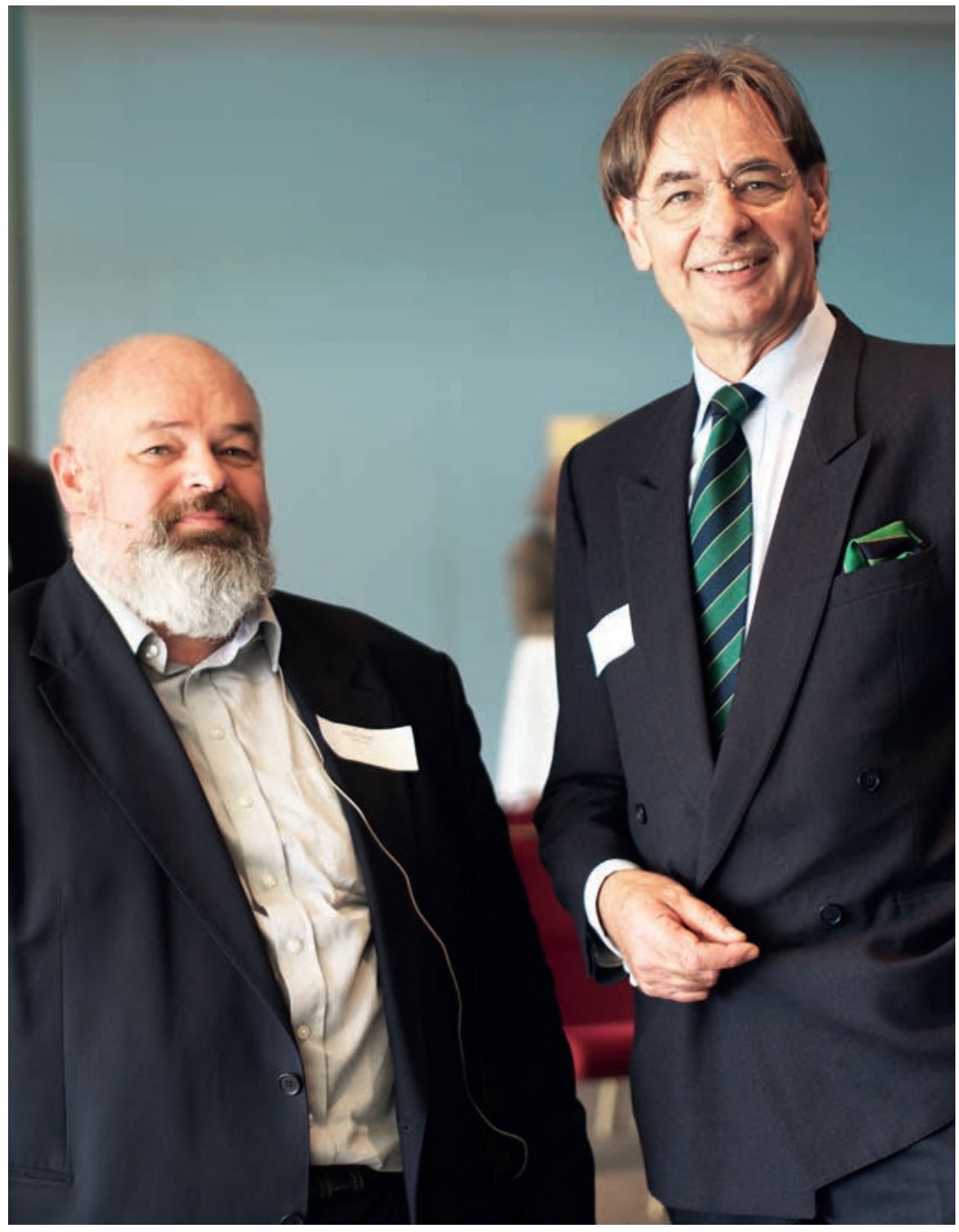

Harry Telser (links) und Josef E. Brandenberg.
Sollen die Kantone die Möglichkeit haben, einen Gesamtbetrag festzulegen, wenn es darum geht, Gesundheitskosten - darunter ambulante ärztliche Leistungen - zu finanzieren? Dies war inhaltlich die Frage, die der Diskussion in der zuständigen Nationalratskommission zugrunde lag. Anfang Februar beschloss sie als Antwort ein «Ja».

Was dafür gesprochen hatte, konnte am fmCh-Podium nicht einmal Heinz Brand sagen, Kommissionsmitglied, santésuisse-Präsident - und Mitglied der SVP, aus deren Parteikreisen der Vorstoss in der SGK-N stammt. «Wahrscheinlich wollte die Kommission ein Zeichen setzen, ein Signal aussenden", mutmasste er in Luzern.

\section{Juristische Bedenken}

Aus rechtlicher Sicht zerzauste Rechtsanwalt Andreas Faller, ehemaliger Leiter des Bereichs Kranken- und Unfallversicherung im Bundesamt für Gesundheit BAG, das umstrittene finanzielle Steuerungsinstrument. Er sprach von Kollisionsfeldern und knirschenden Reibeflächen, und er zitierte als grundlegendes Gegenargument die Bundesverfassung: Staatliches Handeln müsse im öffentlichen Interesse liegen und verhältnismässig sein, sagte er - und dies sei hier nicht der Fall. Auch liege ein Globalbudget in einem eklatanten Widerspruch zu grundsätzlichen Prinzipien wie Rechtsgleichheit und Wirtschaftsfreiheit. «Die Sippenhaft ist ein Rechtsgrundsatz aus dem Mittelalter», sagte Faller. Und: «Deutschland hat's - aber bei uns ist diese Vorlage trotzdem nicht rechtskonform.» 


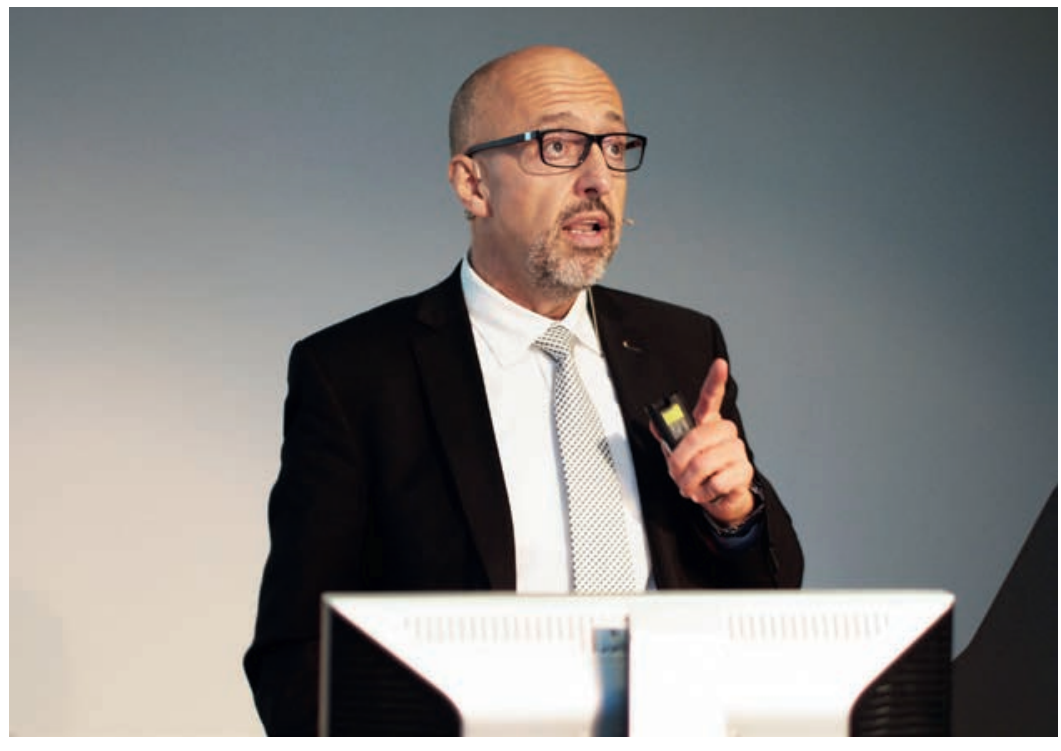

Andreas Faller. sondern Geldströme zu lenken», sagte Schulte, «ärztlichen Korporatismus zu brechen, indem man konkurrierende Interessen und zirkulierende partikuläre Vorteilsnahmen schafft.» Womit er explizit die Versicherungen im Visier hatte: "Sie wollen schlechte Risiken draussen haben - eine Lachnummer.»

Aber auch die Folgen für die Ärzteschaft beleuchtete Schulte. Einzelne Praxen müssten «ein Budget abarbeiten», prophezeite er. «Dies führt zu Nonsens-Untersuchungen und langen Wartezeiten für die Patienten.» Kanada kenne das System seit Anfang neunziger Jahre, und von dort wisse man, dass die Ärzte wegen des wirtschaftlichen Drucks tatsächlich begonnen hätten, mehr Leistungen zu erbringen, berichtete der Ökonom Dr. Harry Telser. «Das Globalbudget ist ein Extrem, bei dem die Leistungserbringer praktisch alleine das Risiko tragen", sagte er. Es biete deshalb auch Anreize zur Patientenselektion, und dies vor allem auf Kosten von chronisch Kranken.

«Wir Ärzte wollen die Patienten nicht krank machen, ich bin nicht daran interessiert, Diagnosen zu produzieren", erklärte in der anschliessenden Diskussion HNO-Spezialist Prof. Dr. med. Marcus Maassen. Ein Globalbudget im Gesundheitswesen aber wäre «die schädlichste Veränderung der letzten Jahre». Da würde «eine fremde Macht der Ärzteschaft das Grab schaufeln», prophezeite er - «und bei den Patienten unwiderrufliche Schäden anrichten».

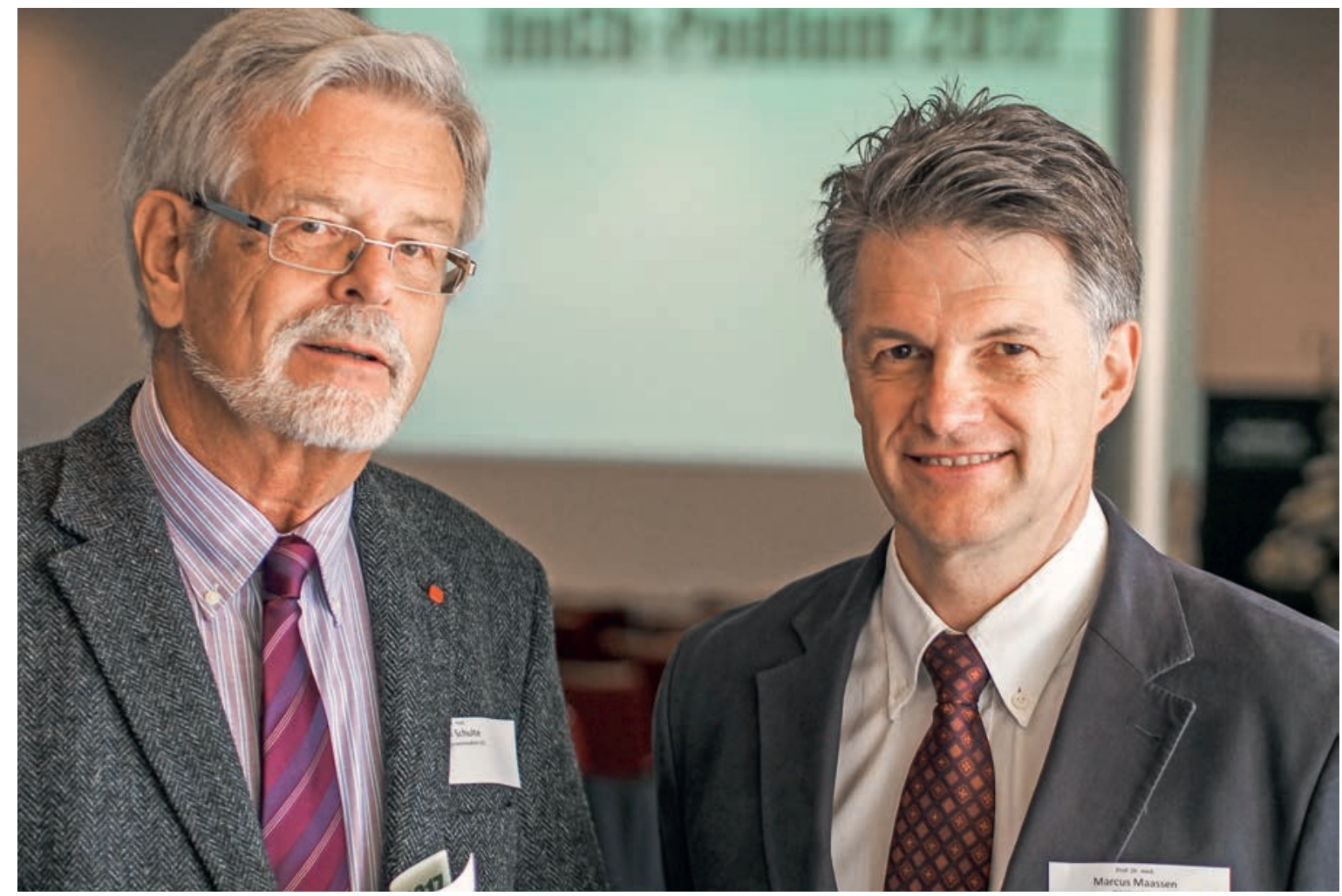

Udo Schulte (links) und Marcus Maassen. 


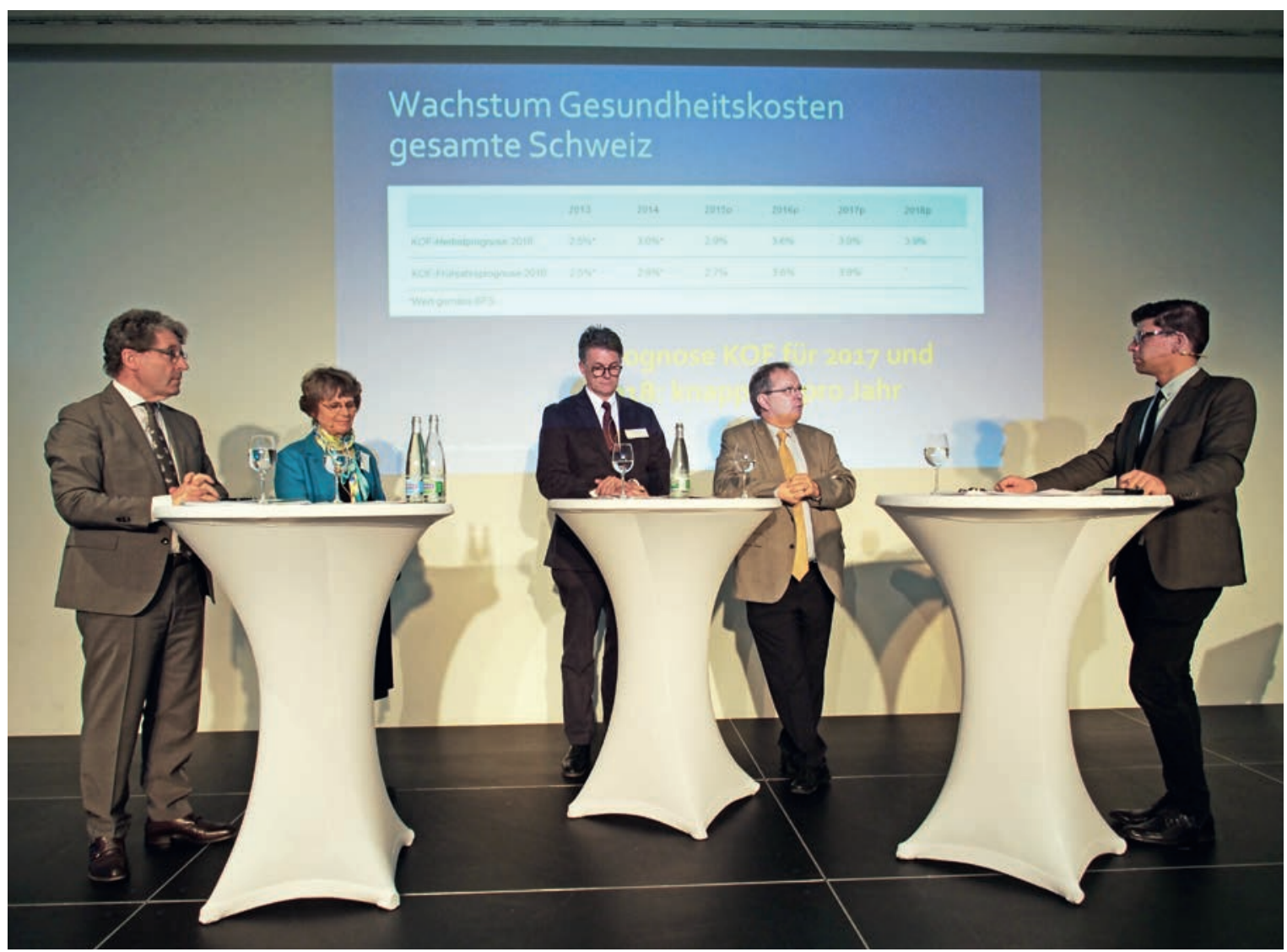

Die Diskussionsrunde (von links): Heinz Brand, Margrit Kessler, Marcus Maassen, Guido Schüpfer und Moderator Jérôme Martinu.

\section{«Ein bürokratisches Monster»}

In die gleiche Kerbe schlug Dr. med. Guido Schüpfer, Stabschef Medizin am Luzerner Kantonsspital. Er sprach von einem «bürokratischen Monster» und wandte sich vor allem gegen «die knallharten Lobbyisten im Parlament, die ihre Töpfe schützen» und "von Regulierungswahn befallen» seien. Im eidgenössischen Parlament habe es «dreimal mehr Spitalvertreter als Krankenversicherer", entgegnete SVP-Nationalrat Brand. Im Übrigen müssten mit Blick auf die Kostenexplosion auch die Patientinnen und Patienten erwähnt werden: «Sie wollen immer mehr Leistungen.» Patientenschützerin und alt Nationalrätin Margrit Kessler nahm stattdessen das politische System ins Visier und forderte für die föderalistische Schweiz "fünf Gesundheitsregionen statt 26 verschiedene Gesundheitswesen».

Wie gesagt: Während der ganzen Veranstaltung im Luzerner Casino war kein einziges positives Argument für die Idee "Globalbudget im Gesundheitswesen" zu vernehmen, auch in der Diskussion nicht, die von Jérôme Martinu, Chefredaktor der Luzerner Zeitung, moderiert wurde. Nationalrat Brand hatte immerhin einen kleinen Trost auf Lager: «Ein Vorstoss allein macht noch keinen Systemwandel», relativierte er das Ja seiner Kommission. Der Weg sei noch lang - und der Ständerat als nächste Hürde eine andere Institution.

Bildnachweis

Fotos: Daniel Lüthi 